\title{
Quark masses from low-energy moments of heavy-quark current correlators
}

\author{
Peter Marquard*† \\ Institut für Theoretische Teilchnphysik \\ Universität Karlsruhe, KIT \\ E-mail: peter.marquardekit.edu
}

We present an update of the determination of charm- and bottom-quark masses from the lowenergy expansion of the vacuum polarization function in combination with experimental data for $R(s)$. This analysis leads to the most precise determination of the masses of the charm and bottom quarks: $m_{c}(3 \mathrm{GeV})=986(13) \mathrm{MeV}, m_{b}\left(m_{b}\right)=4163(16) \mathrm{MeV}$.

European Physical Society Europhysics Conference on High Energy Physics, EPS-HEP 2009,

July 16 - 222009

Krakow, Poland

\footnotetext{
* Speaker.

${ }^{\dagger}$ In collaboration with K. Chetyrkin, Y. Kiyo, J.H. Kühn, A. Maier, P. Maierhöfer, A.V. Smirnov, M. Steinhauser, C. Sturm
} 


\section{Introduction}

The precise determination of the masses of the charm and bottom quark are of utmost importance since many observables in flavor physics are highly sensitive to them. Important examples are the semi-leptonic decay rates of $B_{d}$ and $B_{c}$ mesons

$$
\Gamma\left(B_{d} \rightarrow \ell v K\right) \propto m_{b}^{5}, \quad \Gamma\left(B_{c} \rightarrow \ell v K\right) \propto m_{b}^{5} f\left(m_{c}^{2} / m_{b}^{2}\right) .
$$

Another example is the decay rate of the Higgs boson into bottom quarks where the dominant uncertainty stems from the dependence on the bottom-quark mass.

$$
\Gamma(H \rightarrow b \bar{b})=\frac{G_{F} M_{H}}{4 \sqrt{2} \pi} m_{b}^{2}\left(1+5.6667 a_{s}+\cdots-825.7 a_{s}^{4}\right), \quad a_{s}=\alpha_{s} / \pi .
$$

One possible and maybe the most precise way to determine the masses of the charm and the bottom quark are low-energy sum rules. Here one compares weighted integrals $\mathscr{M}_{n}^{\text {exp }}$ of the experimentally measured R-ratio

$$
\mathscr{M}_{n}=\int d s \frac{R(s)}{s^{n+1}}, \quad \quad R(s)=\frac{\sigma\left(e^{+} e^{-} \rightarrow \text { hadrons }\right)}{e^{+} e^{-} \rightarrow \mu^{+} \mu^{-}}
$$

on the one hand and coefficients $C_{n}^{\text {th }}$ of the low-energy expansion of the vacuum polarization function

$$
\Pi^{\mu v}\left(q^{2}\right)=\left(q^{2} g^{\mu v}-q^{\mu} q^{v}\right) \Pi\left(q^{2}\right), \quad \Pi\left(q^{2}\right)=Q_{q}^{2} \frac{3}{16 \pi^{2}} \sum C_{n}^{\text {th }} z^{n}, \quad z=\frac{q^{2}}{4 m_{q}^{2}}
$$

on the other hand. Relating Eq. (1.3) and Eq. (1.4) by means of a dispersion relation the quark mass is given by

$$
m_{q}=\frac{1}{2}\left(\frac{9 Q_{q}^{2} C_{n}^{\mathrm{th}}}{4 \mathscr{M}_{n}}\right)^{\frac{1}{2 n}} .
$$

In the following we will discuss how to calculate the low-energy expansion of the vacuum polarization function and use it in combination with experimental data for the R-ratio in order to determine the values of the quark masses.

\section{Low-energy expansion of the vacuum polarization function and reconstruction of $\mathbf{R}(\mathbf{s})$ using Padé approximations}

The low-energy expansion of the vacuum polarization has been computed up to four-loops in perturbative QCD $[1,2,3,4,5]$. While at four-loops it was only possible to calculate the first three terms, at three-loop level as many as 30 terms are available $[6,7]$. In the four-loop case the limiting factor is the factorial growth of the number of integrals appearing in the calculation. The details of the calculation are beyond the scope of this article, so we only give the numerical results in Table 1.

Besides the terms in the low-energy expansion discussed above much more information is available for the behavior of the vacuum polarization function. There are two more distinct energy regions, namely the euclidean high-energy and the threshold region. In both regions some terms in 


\begin{tabular}{c|c|c|c}
\hline & $n_{l}=3$ & $n_{l}=4$ & $n_{l}=5$ \\
\hline \hline$C_{1}^{(3)}$ & -5.6404 & -7.7624 & -9.6923 \\
$C_{2}^{(3)}$ & -3.4937 & -2.6438 & -1.8258 \\
$C_{3}^{(3)}$ & -2.8395 & -1.1745 & 0.4113 \\
$C_{4}^{(3)}$ & $-3.349(11)$ & $-1.386(10)$ & $0.471(9)$ \\
$C_{5}^{(3)}$ & $-3.737(32)$ & $-1.754(32)$ & $0.104(27)$ \\
$C_{6}^{(3)}$ & $-3.735(61)$ & $-1.910(63)$ & $-0.228(54)$ \\
$C_{7}^{(3)}$ & $-3.39(10)$ & $-1.85(10)$ & $-0.46(9)$ \\
$C_{8}^{(3)}$ & $-2.85(13)$ & $-1.67(14)$ & $-0.66(12)$ \\
$C_{9}^{(3)}$ & $-2.22(17)$ & $-1.47(18)$ & $-0.91(16)$ \\
$C_{10}^{(3)}$ & $-1.65(20)$ & $-1.37(22)$ & $-1.30(19)$ \\
\hline
\end{tabular}

Table 1: Low-energy expansion coefficients of the vector current correlator in four-loop approximation. The heavy quark mass is renormalized in the $\overline{\mathrm{MS}}$ scheme. The number of light quarks is given by $n_{l}$. The first three coefficients have been calculated directly while the others are obtained from the reconstructed form of the vacuum polarization function.
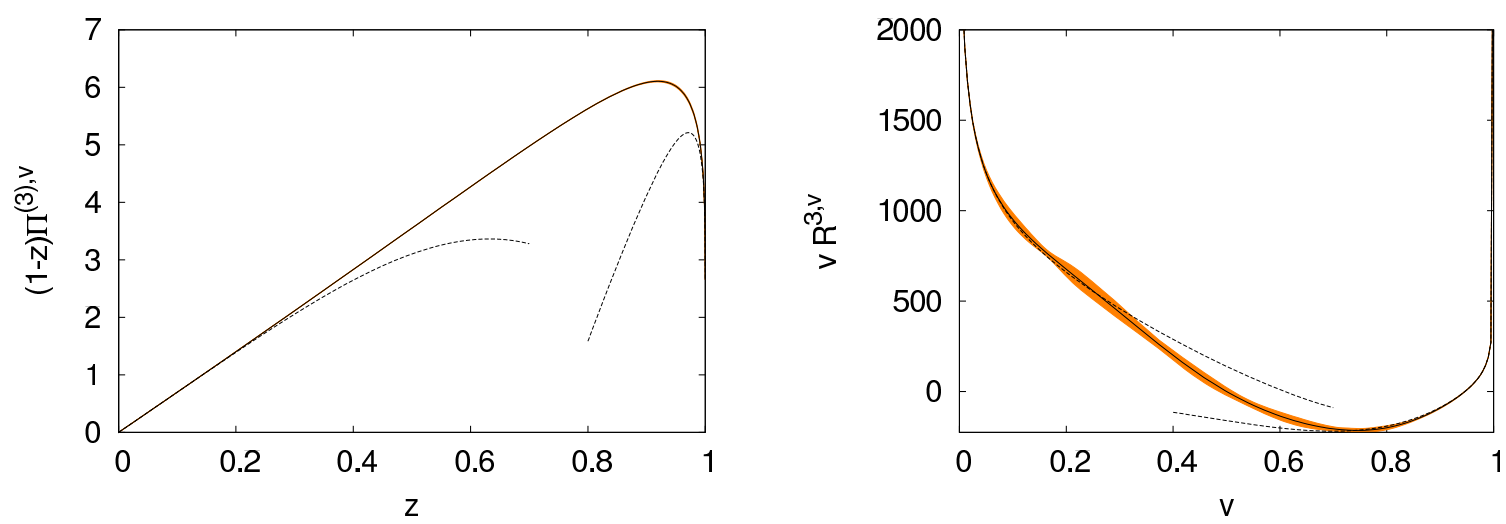

Figure 1: Padé approximations of the vacuum polarization function below threshold (left) and the $R$-ratio above threshold (right). In addition, the expansions about $q^{2}=0, q^{2}=4 m^{2}$ and $q^{2} \rightarrow \infty$ are shown. The $R$-ratio is plotted against the velocity of the heavy quarks.

the corresponding expansions are known. Combining all the available information one can try to reconstruct the full energy dependence of the vacuum polarization function [8,9]. This approach relies on the construction of Padé approximants, which are a generalization of the well known Taylor series. Once the full energy dependence of the vacuum polarization function is known it can be used to obtain precise predictions for the coefficients of the low-energy expansion. These predictions are given in Table 1. Examples for the form of vacuum polarization function are shown in Fig. 1. There we show its behavior below threshold, while above threshold we show $R(s)$ which can be related to the vacuum polarization function using $R(s)=12 \pi \operatorname{Im}(\Pi(s+i \varepsilon))$.

\section{Determination of the Quark Masses}

The main experimental ingredient is the measurement of $R(s)$ at the threshold region. In case of the charm quark the available data is in good agreement with the predictions of perturbative QCD 


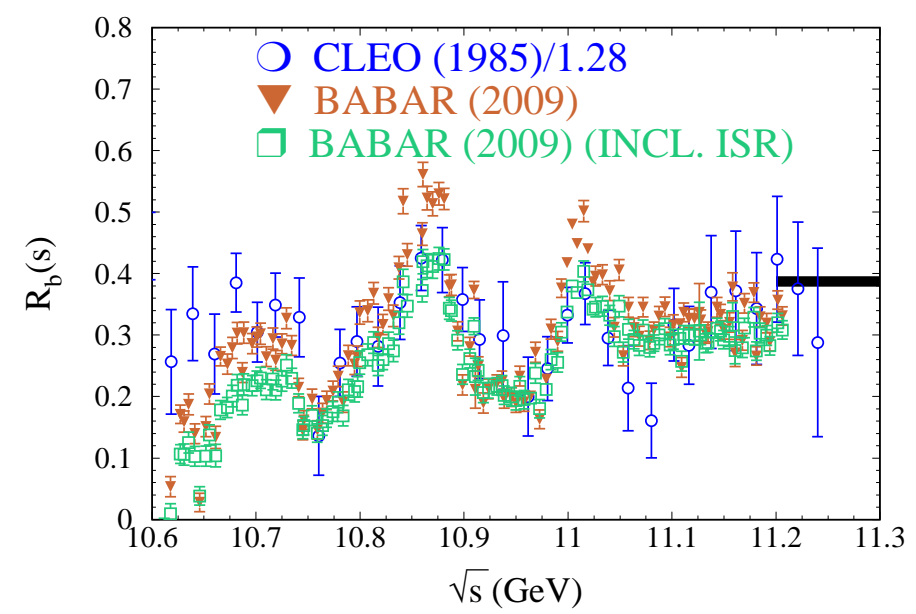

Figure 2: $R(s)$ below the $b \bar{b}$ threshold. In the figure the rescaled CLEO data is shown in comparison with the new data from the BABAR collaboration.

which is valid above threshold. The analysis leads to a value of $m_{c}(3 \mathrm{GeV})=986(13) \mathrm{MeV}$, which together with the analysis in [10] based on lattice simulations constitutes the most precise determination of the charm-quark mass. In the case of the bottom quark, the situation was less clear for quite some time since the CLEO data from 1985 was clearly in disagreement with the predictions from perturbative QCD and was therefore rescaled to match these predictions. The situation has greatly been improved with new data from the BABAR collaboration (cp. Fig. 2). Incorporating the new data in the analysis for the bottom-quark mass and using the improved predictions for the theoretical low-energy parameters shown above one obtains a value of $m_{b}\left(m_{b}\right)=4163(16) \mathrm{MeV}$ for the mass of the bottom quark. The full details of the analysis can be found in [11].

\section{References}

[1] K. G. Chetyrkin, J. H. Kühn, C. Sturm Eur. Phys. J., C48:107-110, 2006.

[2] R. Boughezal, M. Czakon, T. Schutzmeier Phys. Rev., D74:074006, 2006.

[3] A. Maier, P. Maierhöfer, P. Marquard, Phys. Lett. B 669 (2008) 88.

[4] C. Sturm, JHEP 0809, 075 (2008).

[5] A. Maier, P. Maierhöfer, P. Marquard, A. V. Smirnov, arXiv 0907.2117.

[6] A. Maier, P. Maierhöfer, P. Marquard Nucl. Phys., B797:218-242, 2008.

[7] R. Boughezal, M. Czakon, T. Schutzmeier Nucl. Phys. Proc. Suppl., 160:160-164, 2006.

[8] A. H. Hoang, V. Mateu, S. Mohammad Zebarjad, Nucl. Phys. B 813 (2009) 349.

[9] Y. Kiyo, A. Maier, P. Maierhöfer, P. Marquard Nucl. Phys. B 823 (2009) 269.

[10] I. Allison et al. [HPQCD Collaboration], Phys. Rev. D 78 (2008) 054513.

[11] K. G. Chetyrkin, J. H. Kühn, A. Maier, P. Maierhofer, P. Marquard, M. Steinhauser and C. Sturm, arXiv:0907.2110 [hep-ph]. 